\title{
Editorial zum Themenschwerpunkt Zugänge bei der Knieendoprothetik
}

In den letzten Jahren hat das Interesse der Chirurgen, der Patienten sowie der Industrie an der minimalinvasiven Implantation von Endoprothesen kontinuierlich zugenommen. Sowohl für die Hüft- als auch die Knieendoprothetik wurden hierfür spezielle Haken und Instrumente entwickelt, die es ermöglichen sollen, die Implantation der Endoprothese bei kleinerem Zugangsweg gleichbleibend sicher durchführen zu können.

Bis vor wenigen Jahren wurde das Ergebnis einer Knieendoprothesenimplantation hauptsächlich an der mit der verwendeten Technik und Prothese einhergehenden Standzeit und Revisionsrate und weniger an der Schnelligkeit der Erreichung funk tioneller Ziele gemessen. Als Konsequenz dieser Betrachtung wurden große Zugänge zur optimalen Visualisierung der gesamten anatomischen Strukturen gewählt, damit die Ziele der Operation mit sicherer Primärfixation und korrekter Positionierung der Implantate, korrekten Achsausrichtungen, guter Beweglichkeit und stabiler Bandführung möglichst sicher erreicht werden konnten.

Die minimalinvasive Knieendoprothetik hat das Spektrum der zu erreichenden Ziele bei und mit der Operation erweitert. Obwohl die langfristigen Ziele bei der minimalinvasiven Chirurgie unverändert bleiben, rücken die kurzfristigen Ziele nach der Operation zusätzlich in den Fokus. Das Konzept der minimalinvasiven Chirurgie besteht darin, bei der Knieendoprothe- tik die Operation möglichst schonend für die das Knie umgebenden Weichgewebe, und hier vor allem die Muskeln, durchzuführen, um eine möglichst rasche Rehabilitation des Patienten zu erzielen und die Dauer des Krankenhausaufenthalts zu reduzieren. Die bisher in der Literatur berichteten Ergebnisse mit verschiedenen minimalinvasiven Implantationstechniken von Knieendoprothesen scheinen diese Hoffnungen zu bestätigen.

Unter den verschiedenen minimalinvasiven Zugangswegen haben sich drei für die Knieendoprothetik bewährt: der Mini-Midvastus-, der Mini-Subvastus- und der Mini-Quadsparing-Zugang. Diese sollen mit ihren Vor- und eventuellen Nachteilen im vorliegenden Schwerpunktheft von auf diesem Gebiet erfahrenen Kollegen dargestellt werden. Das oberste Ziel muss jedoch nach wie vor die sichere und korrekte Implantation der Knieendoprothese bleiben, so dass jeder einzelne minimalinvasive Zugang auch hinsichtlich der einfachen Erweiterungsmöglichkeit bei intraoperativen Problemen beurteilt werden muss.

Gemeinsam ist den minimalinvasiven Techniken, dass sie eine gewisse Lernkurve für den Chirurgen mit sich bringen und daher nur von erfahrenen Kollegen, die die Implantation einer Knieendoprothese über einen normalen Standardzugang reproduzierbar sicher beherrschen, angewandt werden sollten.

Bernd Fink
Oper Orthop Traumatol 2009;21:1

DOI 10.1007/s00064-009-1600-3 